\title{
White Dwarfs are Small, Fast-Spinning Hot Stars
}

\author{
Weitter Duckss ${ }^{1}$
}

\author{
${ }^{1}$ Independent Researcher, Zadar, Croatia \\ Projetc: https://www.svemir-ipaksevrti.com/
}

\begin{abstract}
Summary: In order to determine the density of white dwarfs and other stars I used a database and created several relations, such as mass/volume of different star types, to create comparable dana, the values of rotation, the percentage of the objects orbiting around a central object and the explanation how different speeds of rotation, if unused, influence the irregular derivation of the gravitational results. Some other factors, essential in creating real values in astrophysics, are also analyzed here. The results acquired in such a way reveal a real image, which is impossible to perceive if analysing only a small or limited quantity of stars and other objects. It doesn't work without a larger sequence of relations of different parameters. The research represents the interweaving of data for stars when indicators start displaying comparable results. The rotation speed value is closely related to star types, as presented in the tables 4 and 6 . At the same time it defines the temperature level of an object, but only faintly affects its density. Density mildly decreases with the increase of the rotation speed, but magnetic field value increases strongly.
\end{abstract}

Keywords: White Dwarfs, Hot Stars, Rotation Speed, Density

\section{Introduction}

The article analyses several parameters, included in several relations, based on which real data representing white dwarfs could be created, in the terms of their real density and some other factors that ascribe white dwarfs into that type of the celestial objects.

Star types are related to the speed of rotation around an object, in the relation with temperature. The influence of rotation is on the magnetic field value, on the percentage of objects in the orbit and on the orbital speeds. Tables 3, 7, 8 and 9 show that objects with the same mass can be classified into groups of many star types. If the effects of the star rotation are ruled out, then a proper answer for such an outcome is not possible to find, because a similar quantity of mass has to produce similar values.

There are more than 270 links in 14 tables, leading towards the database, in which a reader can check the source of information (reference). The goal of this is not to dispute or to support the mainstream points of view, but to introduce real data checking, which is available these days in the form of the official scientific measuring. The topic on matter is not limited to white dwarfs, but it rather analyzes all star types and the centers of galaxies.
2. Determining the density of white dwarfs and "normal" stars

2.1. Determining the density of white dwarfs and "normal" hot stars

I use the existing databases in providing evidence to support or dispute the existence of extreme densities of stars and other objects. All evidence are related to the source of information through one or several steps. [1]

The method to acquire reliable data is to create a sequence of relations from the official measuring results, carried out and obtained on the same place and without the possibility to manipulate the results. The selection of evidence to be analyzed is as it is, because generally there are no cumulative data (temperature, mass, radius, luminosity, etc.) for a large number of objects which are used for relation sequences, in order to analyze matter from all angles. A part of the evidence are here on purpose, to be relevant and comparable inside the relations. The data from the relations are intended to cover the whole diapason of values: mass, radius, temperature, etc. A single object of a certain type is never an object of analysis, not even in a single case. If based on particular cases, the conclusions tend to be opposite to the real situation. 
Table 1. The observation of the parallel indicators of mass, radius, temperature and surface gravity

\begin{tabular}{|c|c|c|c|c|c|}
\hline Star & Volume & Mass, Sun=1 & Radius, Sun=1 & Mass/volume & Type of star \\
\hline V391 Pegasi & 0,02865 & $0,5 \pm 0,05$ & $0,23 \pm 0,03$ & 17,45 & blue-white subdwarf star \\
\hline HD_49798 & 7,1795 & 1,5 & 1,45 & 0,2089282 & sdO6p \\
\hline NSVS 14256825 & 0,016153 & 0,528 & 0,19 & 32,687 & sdOB / M V \\
\hline $2 M A S S J 19383260+4603591$ & $0,026 / 0,0093$ & $0,48 / 0,120$ & $0,223 / 0,158$ & $18,46 / 12,9$ & $\mathrm{sdBV} / \mathrm{M}$ \\
\hline$H V S 7$ & 150,72 & 3,7 & 4,0 & 0,02455 & $\mathrm{sdB}$ \\
\hline Kepler-70 & 0,0197 & 0,496 & 0,203 & 25,178 & $\mathrm{sdB}$ \\
\hline$P G 1047+003$ & 0,07948 & 0,5 & 0,15 & 62,91 & $\mathrm{sdBe}$ \\
\hline Groombridge 1830 & 0,744 & 0,661 & 0,681 & 0,8884 & class G8 subdwarf \\
\hline Kapteyn's Star & 0,058 & 0,274 & 0,291 & 4,7241 & sdM1 \\
\hline HD 134439 & 0,4431 & $\sim 0,78$ & 0,573 & 1,76 & sd:K1Fe-1 \\
\hline HD 134440 & 0,3596 & $\sim 0,73$ & 0,5345 & 2,03 & $\mathrm{sdK} 2.5$ \\
\hline Sun $(M=1, R=1)$ & 2,355 & 1 & 1 & $0,4246$. & G2V \\
\hline WR 102 & 0,33113184 & 16,7 & 0,52 & 50,433 & WR- WO2 \\
\hline WR $93 b$ & 0,20061 & 8,1 & 0,44 & 40,377 & WR_WO3 \\
\hline WR 142 & 1,2058 & 28,6 & 0,8 & 23,72 & WR- WO2 \\
\hline$W R 7$ & 4,711 & 13 & 1,26 & 2,76 & WR-WN4-s \\
\hline$W R 46$ & 5,924 & 14 & 1,36 & 2,2633 & WR- WN3p-w \\
\hline WR 3 & 35,921 & 15 & 2,48 & 0,4176 & WR-WN3-hw \\
\hline WR 21a & 4069,44 & 103,6 & 12 & 0,02546 & WR-WN5ha \\
\hline WR $31 a$ & $62.231,76$ & 17 & 29,8 & 0,0002732 & WR-WN11h \\
\hline Lambda Cephei & $17.462,0$ & 51 & $18-21$ & 0,00292 & O6.5If(n)p \\
\hline NML Cygni & 3'898'927.371,9 & 50 & $1.183,0$ & 0,000000013 & M4.5-M7.9Ia-III \\
\hline $\operatorname{Ros} 47$ & 0,01157 & 0,35 & 0,17 & 30,1724 & $\mathrm{M} 4.0 \mathrm{Vn}$ \\
\hline Kepler-42 & 0,01157 & 0,13 & 0,17 & 11,236 & M5V \\
\hline YZ Canis Minoris & 0,0801 & 0,308 & 0,324 & 3,845 & M5V \\
\hline LHS 1140 & 0,015154 & 0,146 & 0,186 & 9,63442 & $\mathrm{M} 4.5 \mathrm{~V}$ \\
\hline SU Ursae Majoris & 0,01097 & 0,105 & 0,167 & 9,572 & dwarf nova \\
\hline OTS 44 & 0,129 & 0,011 & $0,23-0,57$ & 0,08527 & r. planet/ Brown Dwarf \\
\hline TVLM 513-46546 & 0,0031345 & 0,09 & 0,11 & 28,7127 & Red/Brown Dwarf-M9 \\
\hline$D E N 0255-4700$ & 0,001716795 & $0,025-0,065$ & $0,08-0,1$ & 26,212 & Brown Dwarf-L8/L9 \\
\hline$O G L E-T R-123$ & 0,00517395 & 0,085 & 0,13 & 16,4285 & Brown Dwarf-M \\
\hline
\end{tabular}

Mass and radius of Jupiter (Jup = 1), density: Sun=1,408 g/ $\mathrm{cm}^{3}$; Jupiter 1,326 g/ $/ \mathrm{cm}^{3}$

\begin{tabular}{|c|c|c|c|c|c|}
\hline Star & Volume & Mass Jup & Radius Jup & Mass/volume & Type of star \\
\hline Teide 1 & 127,1939 & $57 \pm 15$ & 3,78 & 0,445 & Brown Dwarf-M8 \\
\hline Cha 110913-773444 & 13,73436 & $8(+7,-3),(17)$ & 1,8 & 0,5825 & r. planet/ Brown Dwarf \\
\hline PSO J318.5-22 12 & 8,4346 & 6,5 & 1,53 & 0,771 & rogue planet \\
\hline $2 M A S S$ J0523-1403 & 2,42636 & 67,54 & 1,01 & 27,84 & Brown Dwarf-L2.5V \\
\hline EBLM J0555-57 Ab & 1,396 & $85,2(\sim 0,081$ Sun $)$ & 0,84 & 61,03 & Brown Dwarf \\
\hline $2 M A S S 0939-2448$ & 1,20576 & $20-50(35)$ & 0,8 & 29,0273 & Brown Dwarf-T8 \\
\hline 15 Sagittae & 2,350 & 68,7 & 1 & 29,172 & Brown Dwarf-L4 \\
\hline LHS $6343 c$ & 1,13051 & 62,1 & 0,783 & 54,931 & Brown Dwarf-T \\
\hline
\end{tabular}

\begin{tabular}{|c|c|c|c|c|c|}
\hline Star & Distance AU & Mass Jup & Radius Jup & Temperature K & Type planet \\
\hline \multicolumn{6}{|c|}{ Srars generate their own energy. Planet reflected radiation, do not create their own energy. } \\
\hline $2 M A S S J 2126-8140$ & $6.900,0$ & 13,3 & I & $1.800,0$ & Planet \\
\hline$R O X s 42 B b$ & 140 & 9 & $0,9-3$ & $1.800,0-2.600,0$ & Planet \\
\hline$H I P 65426 b$ & 92 & 9 & 1,5 & $1.450,0$ & Planet \\
\hline$H R 8799 d$ & 24 & 7 & 1,2 & $1.090,0$ & Planet; density $4 \mathrm{~kg} \mathrm{~m}^{3}$ \\
\hline$H R 8799 c$ & 38 & 7 & 1,3 & $1.090,0$ & Planet; density $3,2 \mathrm{~kg} \mathrm{~m}^{3}$ \\
\hline DH Таи B & 330 & 11 & 2,7 & $2.750,0$ & Planet \\
\hline UScoCTIO $108 b$ & 670 & 14 & 0,9078 & $2.350,0$ & Planet \\
\hline $11 O p h b$ & 472,9 & 21 & 0,9078 & $2.375,0$ & Planet \\
\hline
\end{tabular}

Table 1. Relationshift: Mass/volume, temperature and surface gravity

The analysis of the objects' density in Table 1 (in the relation of mass/volume - star type) points out that there is no consistency that would be related to star types. Inside a same star type there are densities, which are lower, higher or the same as the one of Sun. The old concept's contours are clearly visible in the statements that smaller stars have higher densities and big red stars are inflated objects. [2] However, 
that concept also lacks consistency. It is particularly important to point out that the mass and radius estimates of the objects that are smaller than the mass and radius of Sun are generally only hypothesized (using the old hypotheses). [3] If a star has the same mass or radius as Sun, the estimate of its density may follow several different hypotheses. For example, if an object is classified into a type of "planets", it is less dense than a type known as a brown dwarf. Brown Dwarfs masses are 0,035 and 68,7 (2MASS 0939-2448 and 15 Sagittae) and it makes mass/volume ratio of 29,0273 and 29,1720 respectively. At the same time, planets with the distances of 38-6.900,0 AU have mass/volume ratio around 1 (ROXs 42B bø0,6036; 11 Oph b 11,8765). In a particular type of stars, Wolf-Rayet stars, there are stars with mass/volume ratio of 0,0002732 (WR $31 a$ ) to 50,4330 (WR 102). M type stars with large quantities of mass suggest their densities are low, because the effects of their slow rotation don't provide the same results with the objects they are interacted with, to the contrary of faster and fast rotating stars. Generally, the decrease of density is ascribed to the stars with the increase of mass above 1 M Sun (Lambda Cephei M $51 \mathrm{M}_{\text {Sun }}$, M/V 0,00292, T $36.000^{\circ} \mathrm{K} ; \quad N M L$ Cygni M $51 \quad \mathrm{M}_{\text {Sun }}, \quad \mathrm{M} / \mathrm{V}$ 0,000000013 , T $\left.2.500-3.250^{\circ} \mathrm{K}\right)$.

Table 2. Density/temperature

\begin{tabular}{llll}
\hline Depth km Earth (Sun) & Component layer & Density g/cm & Temperature K \\
\hline $0-35$ & Crust & $2,2-2,9$ & -86 to 200 (400) \\
\hline $35-2.890$ & Mantle & $3,4-5,6$ & $200-4.000$ \\
\hline $5.100-6.378$ & Inner core & $12,8-13,1$ & $5.400-5.700(6.000)$ \\
\hline$>520.000,0$ Sun & Sun core & 150 & 15,7 million
\end{tabular}

Table 2. Depth km /Density/temperature

The temperature and density increases with depth. White dwarf temperatures do not follow this basic law. Their recommended density of $31.000,0$ to above 460.000,0 (1'000.000-1'500.000) g/ $\mathrm{cm}^{3}$ would generate temperatures above 100 billion K.
Temperatures white dwarfs are from under 10.000 $\left(4.270 \pm 70\right.$ Gliese $223.2 ;$ G $\left.240-725.590,0 \pm 90^{\circ} \mathrm{K}\right)$ to $200.000^{\circ} \mathrm{K} ;\left(H 1504+65,200.000^{\circ} \mathrm{K} ; 310.000{ }^{\circ} \mathrm{K}\right.$ PSR B0943 + 10) [6] like normal hot stars.

Table 3. Small stars/ temperature and type of star

\begin{tabular}{|c|c|c|c|}
\hline Small star & Mass Sun=1 & Temper. K & Type \\
\hline Beta Pictoris $b$ & $0,0086-0,012$ & $1.724,0$ & Exoplanet, dist. $11,8 \mathrm{AU}$ \\
\hline CW Leonis & $0,7-0,9$ & $2.000,0$ & $\mathrm{C} 9,5 \mathrm{e}$ \\
\hline Kelu-1 & 0,060 & 2.020 & brown dwarfs L2 \\
\hline Gliese 570 & 0,55 & 2.700 & M1V \\
\hline HIP $78530 \mathrm{~b}$ & 0,022 & 2.800 & exoplanet; dist. $710 \mathrm{AU}$ \\
\hline Castor $C$ & 0,5992 & 3.820 & BY Draconis dwarf stars \\
\hline HIP 12961 & 0,63 & $3.838,0$ & red dwarf M0V \\
\hline$L P 658-2$ & $0,45(0,80)$ & $4.270(5.180)$ & white dwarf DZ11.8 \\
\hline$H R 9038 A b$ & 0,67 & $4.620,0$ & red dwarf $\mathrm{K} 3 \mathrm{~V}$ \\
\hline Groombridge 1830 & 0,661 & 4.759 & G8 subdwarf \\
\hline S Arae & 0,51 & 6.563 & A3 II \\
\hline HR 4049 & 0,56 & 7.500 & B9.5Ib-II \\
\hline$G D 356$ & 0,67 & 7.510 & white dwarf DC7 \\
\hline Zeta Cygni B & 0,6 & 12.000 & white dwarf DA4.2 \\
\hline 40 Eridani $B$ & 0,573 & 16.500 & white dwarf DA4 \\
\hline Kepler-70 & 0,496 & 27.730 & $\mathrm{sdB}$ \\
\hline V391 Pegasi & 0,5 & $29.300,0$ & blue-white subdwarf star \\
\hline $2 M A S S J 19383260+4603591$ & 0,48 & 29.564 & $\mathrm{sdBV} / \mathrm{M}$ \\
\hline$P G 0112+104$ & $0,52 \pm 0,05$ & $>30.000$ & white dwarf \\
\hline$P G 1047+003$ & $\sim 0,5$ & 33.500 & $\mathrm{sdBe}$ \\
\hline$L S I V-14116$ & 0,485 & 34.950 & sdB0.5VIIHe18 \\
\hline
\end{tabular}

Table 3. Small stars, mass $\sim 0,5$ MSun (except 3 exoplanets and Kelu- 1 ) in relation to temperature and type of stars 
We see here that part of the white dwarfs is not separated from other star types in terms of temperature. The same mass of small stars does not give the same temperature. White dwarfs have low (4.270,0 (5.180) HIP 12961) and high temperatures (PG 0112+104>30.000). The height of these temperatures covers the spectral type stars from $\mathrm{K}$ to O.

\subsection{White Dwarfs vs. other types of stars with an emphasis on the speed of rotation}

Now, let's determine which basic forces give stars different values of temperature, luminosity, the relation of mass/radius and the value of surface gravity.

Table 4. The relation (of the section of main star types) of rotation, mass, radius, temperature and type

Star Speed rotation $\quad$ Maas Sun=1 $\quad$ Radius Sun=1 $\quad$ Temperature K Type

\begin{tabular}{|c|c|c|c|c|c|c|}
\hline White Dwarf & & & & & & \\
\hline GD 356 & 115 & minutes & 0,67 & 1 & $7.510,0$ & white dwarf \\
\hline EX Hydrae & 67 & minutes & $0,55 \pm 0,15$ & I & 1 & white dwarf \\
\hline AR Scorpii A & 1,95 & minutes & $0,81-1,29$ & 1 & 1 & white dwarf pulsar \\
\hline V455 Andromedae & 67,62 & second & 0,6 & I & I & white dwarf \\
\hline$R X$ Andromedae & 200 & $\mathrm{~km} / \mathrm{s}$ & 0,8 & 1 & $40.000-45.000,0$ & white dwarf \\
\hline RX J0648.0-4418 & 13,2 & second & 1,28 & 1 & 1 & white dwarf \\
\hline \multicolumn{7}{|l|}{ Pulsar } \\
\hline PSR J0348+0432 & 39,123 & m. second & $2,01 \pm 0,04$ & $13 \pm 2 \mathrm{~km}$ & 1 & pulsar \\
\hline Vela $X-1$ & 283 & second & 1,88 & $\sim 11,2$ & $31.500,0$ & X-ray pulsar, B-type \\
\hline $\operatorname{Cen} X-3$ & 4,84 & second & $20,5 \pm 0,7$ & 12 & 39.000 & $\mathrm{X}$-ray pulsar \\
\hline PSR B0943 + 10 & 1,1 & second & 0,02 & $2,6 \mathrm{~km}$ & $310.000,0$ & pulsar \\
\hline PSR $1257+12$ & 6,22 & m. second & 1,4 & $10 \mathrm{~km}$ & 28.856 & pulsar \\
\hline \multicolumn{7}{|l|}{ Wolf-Rayet stars } \\
\hline$H D 5980 B$ & $<400$ & $\mathrm{~km} / \mathrm{s}$ & 66 & 22 & 45.000 & WN4 \\
\hline WR 2 & 500 & $\mathrm{~km} / \mathrm{s}$ & 16 & 0,89 & 141.000 & WN2-w \\
\hline WR 142 & $1.000,0$ & $\mathrm{~km} / \mathrm{s}$ & 28,6 & 0,80 & 200.000 & WO2 \\
\hline$R 136 a 2$ & 200 & $\mathrm{~km} / \mathrm{s}$ & 195 & 23,4 & 53.000 & WN5h \\
\hline \multicolumn{7}{|l|}{ Normal hot stars } \\
\hline VFTS 102 & $600 \pm 100$ & $\mathrm{~km} / \mathrm{s}$ & $\sim 25$ & 1 & $36.000 \pm 5.000$ & O9:Vnnne \\
\hline BV Centauri & $500 \pm 100$ & $\mathrm{~km} / \mathrm{s}$ & 1,18 & I & $40.000 \pm 1.000$ & G5-G8IV-V \\
\hline Gamma Cassiopeiae & 432 & $\mathrm{~km} / \mathrm{s}$ & 14,5 & 8,8 & 25.000 & B0.5IVe \\
\hline LQ Andromedae & 300 & $\mathrm{~km} / \mathrm{s}$ & 8,0 & 3,4 & $40.000-44.000$ & O4If(n)p \\
\hline Zeta Puppis & 220 & $\mathrm{~km} / \mathrm{s}$ & $22,5-56,6$ & $14-26$ & $40.000-44.000$ & O4If(n)p \\
\hline LH54-425O5 & 250 & $\mathrm{~km} / \mathrm{s}$ & 28 & 8,1 & 45.000 & $\mathrm{O} 5 \mathrm{~V}$ \\
\hline Melnick 42 & 240 & $\mathrm{~km} / \mathrm{s}$ & 189 & 21,1 & 47.300 & O2If \\
\hline BI 253 & 200 & $\mathrm{~km} / \mathrm{s}$ & 84 & 10,7 & 50.100 & O2V-III(n) $\left(\left(\mathrm{f}^{*}\right)\right)$ \\
\hline \multicolumn{7}{|l|}{ Red Dwarf } \\
\hline Gliese 876 & 96,6 & days & 0,37 & 0,3761 & $3.129 \pm 19$ & M4V \\
\hline Kepler-42 & $2,9 \pm 0,4$ & $\mathrm{~km} / \mathrm{s}$ & $0,13 \pm 0.05$ & $0,17 \pm 0.04$ & $3.068 \pm 174$ & M5V \\
\hline Kapteyn's star & 9,15 & $\mathrm{~km} / \mathrm{s}$ & 0,274 & $0,291 \pm 0.025$ & $3.550 \pm 50$ & sdM1 \\
\hline Wolf 359 & $<3,0$ & $\mathrm{~km} / \mathrm{s}$ & 0,09 & 0,16 & $2.800 \pm 100$ & M6.5 Ve \\
\hline \multicolumn{7}{|l|}{ Normal cool stars } \\
\hline HD 220074 & 3,0 & $\mathrm{~km} / \mathrm{s}$ & $1,2 \pm 0.3$ & $49.7 \pm 9.5$ & $3.935 \pm 110$ & M2III \\
\hline V Hydrae & $11-14$ & $\mathrm{~km} / \mathrm{s}$ & 1,0 & $420-430$ & 2.650 & $\mathrm{C} 6,3 \mathrm{e}$ \\
\hline$\beta$ Pegasi & 9,7 & $\mathrm{~km} / \mathrm{s}$ & 2,1 & 95 & 3.689 & M2.5II-IIIe \\
\hline Betelgeuse & 5 & $\mathrm{~km} / \mathrm{s}$ & 11,6 & $887 \pm 203$ & 3.590 & M1-M2 Ia-ab \\
\hline \multicolumn{7}{|l|}{ F Type Star } \\
\hline Beta Virginis & 4,3 & $\mathrm{~km} / \mathrm{s}$ & 1,25 & $1.681 \pm 0.008$ & $6.132 \pm 26$ & F9 V \\
\hline pi3 Orionis & 17 & $\mathrm{~km} / \mathrm{s}$ & 1,236 & 1,323 & $6.516 \pm 19$ & F6 V \\
\hline 4 Equulei & $6,2 \pm 1,0$ & $\mathrm{~km} / \mathrm{s}$ & 1,39 & $\sim 1,2$ & $6.213 \pm 63$ & F8 V \\
\hline 6 Andromedae & 18 & $\mathrm{~km} / \mathrm{s}$ & 1,30 & 1,50 & $6.425 \pm 218$ & F5 V \\
\hline
\end{tabular}

Table 4. The relation (of the section of main star types) of rotation, mass, radius, temperature and type

A column "Speed rotation" points to very fast rotations of white dwarfs [4], [5], pulsars, Wolf-Rayet stars and O, B type stars.

Small hot stars make a rotation in a very short period (from miliseconds to a few minutes). Large hot stars rotate at the speed of above $400 \mathrm{~km} / \mathrm{s}$ (Gamma Cassiopeiae). White dwarfs with a diameter of $\sim 80$ $\mathrm{km}$ makes a rotation generally in a few seconds $(R X$ J0648.0-4418 13 seconds). [6]

Wolf-Rayet stars are very fast-rotating stars, the speeds of which can be up to $1.000 \mathrm{~km} / \mathrm{s}$, which is generally accompanied by very high temperatures (WR $142200.000^{\circ} \mathrm{K}, 1.000,0 \mathrm{~km} / \mathrm{s}$ ).

With the decrease of the rotational speed there is also the decrease of a star's temperature. Here it needs to be mentioned that 
Quote: Temperature and radiance are also affected by the tidal forces from the bigger or smaller binary effect, environment, the density of gas (layers) between the observer and a star, the speed of outer matter influx to the object, especially into a whirl or cyclone on the poles of a star (over 140 tons of space matter is falling daily to the surface of Earth [16]), different sums of the mass and rotation effects to the small and big stars. [7] end quote

Large (medium and small) red stars have the rotation from +0 to above $10 \mathrm{~km} / \mathrm{s}$ and temperatures of 1.800 to above $4.000^{\circ} \mathrm{K}$ ( $S$ Cassiopeiae $1.800,0 ; W$ Aquilae 1.800; V Hya 2.160; II Lup 2.000; V Cyg 1.875; LL Peg 2.000; LP And 2.040; V384 Per 1.820; S Aur 1.940; QZ Mus 2.200; AFGL 4202 2.200: V821 Her 2.200; V1417 Aq1 2.000; S Cep 2.095; etc.). [8]

A smaller star needs higher speed to achieve temperatures similar to those of large stars and the reason for it is that a larger object has more matter, which by friction and different speeds of rotation of different layers, creates higher temperatures.

Table 5. The relation white dwarfs / other star types within the relation: temperature / age of stars

\begin{tabular}{|c|c|c|c|}
\hline Star & Temperature K & Age Gyr & Type of stars \\
\hline Gliese 876 & $3.129,0 \pm 19$ & $0,1-9,9$ & M4V \\
\hline LHS 1140 & $3.131 \pm 100$ & $>5$ & $\mathrm{M} 4.5 \mathrm{~V}$ \\
\hline Kapteyn's star & $3.550 \pm 50$ & $\sim 11$ & sdM1 \\
\hline WD $0346+246$ & $3.800 \pm 100$ & $11-12$ & white dwarf \\
\hline Castor C & 3.820 & $370 \mathrm{Myr}$ & dM1e \\
\hline G 240-72 & $5.590 \pm 90$ & 5,69 & white dwarf DQP9.0 \\
\hline G99-47 & $5.790 \pm 110$ & 3,97 & white dwarf DAP8.9 \\
\hline V382 Carinae & 5.866 & 6,8 & G0-4-Ia \\
\hline LSPM J0207+3331 & $6.120,0$ & 3 & white dwarf \\
\hline Beta Virginis & $6.132 \pm 26$ & $2,9 \pm 0.3$ & F9 V \\
\hline pi3 Orionis & $6.516 \pm 19$ & 1,4 & F6 V \\
\hline 4 Equulei & $6.213 \pm 63$ & 3,07 & $\mathrm{~F} 8 \mathrm{~V}$ \\
\hline 6 Andromedae & $6.425 \pm 218$ & 2,91 & F5 V \\
\hline GD 356 & $7.510,0$ & 2,1 & white dwarf \\
\hline Ross 640 & 8.100 & 1,2 & white dwarf DZA5.5 \\
\hline Denebola & 8.500 & $100-380 \mathrm{Myr}$ & $\mathrm{A} 3 \mathrm{Va}$ \\
\hline LP 145-141 & $8.500 \pm 300$ & 1,44 & white dwarf DQ6 \\
\hline Gliese 318 & $9.120,0$ & $550 \mathrm{Myr}$ & white dwarf DA5.5 \\
\hline$H D 21389$ & 9.730 & 11 & A0Iae \\
\hline WD 0806-661 & $10.205 \pm 390$ & 0,62 & white dwarf DQ4.2 \\
\hline$\varepsilon$ Reticuli B & $15.310 \pm 350$ & 1,5 & white dwarf \\
\hline$\eta$ Aurigae & 17.201 & $22-55 \mathrm{Myr}$ & B3V \\
\hline GD 61 & 17.280 & $200 \mathrm{Myr}$ & white dwarf DA \\
\hline Sirius $B$ & $25.000 \pm 200$ & $228 \mathrm{Myr}$ & white dwarf DA2 \\
\hline LQ Andromedae & $40.000-44.000$ & 3,4 Myr & O4If(n)p \\
\hline Zeta Puppis & 40.000 & 3,2 $\mathrm{Myr}$ & O4If(n)p \\
\hline LH54-425 O5 & 45.000 & 2,0 Myr & $\mathrm{O} 5 \mathrm{~V}$ \\
\hline Melnick 42 & $47.300,0$ & $\sim 1 \mathrm{Myr}$ & O2If \\
\hline
\end{tabular}

Table 5. The relation white dwarfs / other star types within the relation: temperature / age of stars

By reviewing the relation white dwarfs / other star types within the relation: temperature / age of stars does not find separation of white dwarfs from other stars. White dwarfs are found within the range of $\mathrm{K}$ to $\mathrm{O}$ star type, in terms of the height temperature and

Table 6. The relation temperature $\mathrm{K} /$ rotation speed

\begin{tabular}{lll}
\hline Star & Temperature K & Rotation speed km/s \\
\hline Slowly-rotating stars & & \\
Betelgeuse & $3.590,0$ & 5 \\
Andromeda 8 & $3.616 \pm 22$ & $5 \pm 1$ \\
B Pegasi & 3.689 & 9,7 \\
Aldebaran & 3.910 & 634 day \\
HD 220074 & 3.935 & 3 \\
Beta Ursae Minoris & 4.030 & 8 \\
Arcturus & 4.286 & $2,4 \pm 1,0$ \\
Hamal & 4.480 & 3,44 \\
Iota Draconis & 4.545 & 1,5 \\
Pollux & 4.666 & 2,8 \\
CCyg A & 4.910 & $0,4 \pm 0,5$ \\
Capella & 4.970 & 4,1 \\
The stars with fast and very fast rotations & & \\
Alpha Pegasi & $9.765,0$ & 125 \\
\hline
\end{tabular}

the recommended age of stars. The temperature is directly related to the speed of rotation (with the exclusion of binary systems effects ...). this is shown in Table 4. 


\begin{tabular}{lll} 
& & \\
\hline Eta Ursae Majoris & 16.823 & 150 \\
$\eta$ Aurigae & 17.201 & 95 \\
Spica secondary & $20.900 \pm 800$ & 199 \\
Gamma Cassiopeiae & 25.000 & 432 \\
S Monocerotis & 38.500 & 120 \\
RX Andromedae (WD) & $40.000,0$ & 200 \\
Zeta Puppis & $40.000-44.000$ & 220 \\
HD 93129 & 42.500 & 130 \\
LH54-425 O5 & 45.000 & 250 \\
LH54-425 O3 & 45.000 & 197 \\
HD 5980 B & 45.000 & 400 \\
Melnick 42 & 47.300 & 240 \\
BI 253 & 50.100 & 200 \\
HD 269810 & 52.500 & 173 \\
WR 2 & 141.000 & 500 \\
WR 142 & $200.000,0$ & 1.000 \\
\hline Table 6. The relation: temperature & / rotation speed
\end{tabular}

Table 6. The relation: temperature / rotation speed

This table draws a sharp line between fast and slow rotating stars.

Quote: A star's speed of rotation causes its temperature (its temperature only partially depends on the mass of a star), its radius (ratio: the mass of a star / the radius of a star; Sun $=1$ ), surface gravity and the color of a star. The stars with a slow rotation are "cold" stars (with the exclusion of binary systems effects), independently of the mass of a star and its radius. Their color is red and they are dominant in Universe

(M type of stars, 0,08-0,45 masses of Sun; $\leq 0.7 \mathrm{R}$ of Sun; $2.400-3.700^{\circ} \mathrm{K} ; 76,45 \%$ of the total number of stars in Milky Way (Harvard spectral classification);

all red stars above $0,45 \mathrm{M}$ of Sun are also included here, as well as the largest red (and other) stars in our galaxy). The stars with fast and very fast rotations are mostly present in nebulae, i.e., in the space which is rich with matter. Their total quantity in Milky Way makes $3,85 \%$ (O class $~ 0,00003 \%$ ). [10] end of quote

\subsection{Similar mass of stars it's situated in different classes (type) and different temperatures}

Table 4. can be presented in such a way to create a relation: approximately the same mass/temperature and relate it to a star type. The relation has to show the same results for the same quantity of mass. It is unacceptable to claim that a single quantity of mass abides by several laws of nature or has several states, which would provide different results. The conditions should be almost identical or we are to explain, why a single quantity of mass has different laws of manifestation. The same goes for the claims that stars realize nuclear fission and fusion on the different levels, because there is one and the same quantity of mass on the same place.

Tabele 7. Star type / mass / temperature

\begin{tabular}{|c|c|c|c|c|}
\hline & Star & Type & Mass Sun=1 & Temperature ${ }^{\circ} \mathrm{K}$ \\
\hline 1 & EZ Canis Majoris & WN3-hv & 19 & 89.100 \\
\hline 2 & Centaurus $X-3$ & $\mathrm{O}$ & $20,5 \pm 0,7$ & 39.000 \\
\hline 3 & $\eta$ Canis Majores & $\mathrm{B}$ & 19,19 & 15.000 \\
\hline 4 & $H D 21389$ & A & 19,3 & 9.730 \\
\hline 5 & Kappa Pavonis & $\mathrm{F}$ & $19-25$ & $5.250-6.350$ \\
\hline 6 & V382 Carinae & $\mathrm{G}$ & 20 & 5.866 \\
\hline 7 & SPersei & $\mathrm{M}$ & 20 & $3.000-3.600$ \\
\hline 8 & DH Tauri b & Planet; dist. $330 \mathrm{AU}$ & 12 M Jupiter & 2.750 \\
\hline 9 & $H I P 78530 \mathrm{~b}$ & Planet; dist. $740 \mathrm{AU}$ & 24 M Jup. & $2.700(2.800)$ \\
\hline
\end{tabular}

Table 7. Stars, similar mass (except No 8, 9, ), different classes (type) and temperatures. [7]

It is obvious from the table that the relation of the same mass, different temperatures and the other star type can be met only by the evidence from the table 4 and 6. [7] , [10] The decrease of the rotational speed (with other incoming factors taken into consideration).
This is no exception, but rather a rule, that a majority of the diapason of the star mass, from the smallest to the largest, the stars belong to different types for any quantity of mass.

Table 8. Star type mass $\sim 17 /$ temperature

\begin{tabular}{lllll}
\hline & Star & Type & Mass Sun=1 & Temperature ${ }^{\circ} \mathrm{K}$ \\
\hline 1. & WR 2, & WN4-s & 16 & 141.000 \\
\hline 2. & M Columbae & $\mathrm{O}$ & 16 & 33.000 \\
\hline 3. & Deneb & $\mathrm{A}$ & 19 & 8.525 \\
\hline 3. & Gamma Cassiopeiae & $\mathrm{B}$ & 17 & 25.000 \\
\hline 4. & VY Canis Majoris & $\mathrm{M}$ & 17 & 3.490 \\
\hline
\end{tabular}




\begin{tabular}{lllll}
\hline 5. & DH Tauri $b$ & Planet; dist. $330 \mathrm{AU}$ & 12 M Jupiter & 2.750 \\
\hline 6. & HIP 78530 $b$ & Planet; dist. $\underline{710 A U}$ & 24 M Jup. & $2.700(2.800)$ \\
\hline 7. & NML Cygni & M & 50 & 3.834 \\
\hline
\end{tabular}

Table 8. Star type / mass $~ 17 /$ temperature [10]

Table 9. Star type/mass $\sim 2 /$ temperature and radius

\begin{tabular}{|c|c|c|c|c|}
\hline Star & Type & Mass $(\operatorname{Sun}=1)$ & Temperature $\mathrm{K}$ & Radius (Sun=1) \\
\hline S Pegasi & M5e - M8.5e & $1,4-1,8$ & 2.107 & $459-574$ \\
\hline$R$ Leporis & C7,6e(N6e) & $2,5-5$ & $2.245-2.290$ & $400 \pm 90$ \\
\hline Rho Orionis & K0 III & 2,67 & 4.533 & 25 \\
\hline 29_Orionis & G8IIIFe-0.5 & 2,33 & 4.852 & 10,36 \\
\hline BX_Andromedae & $\mathrm{F} 2 \mathrm{~V}$ & 2,148 & 6.650 & 2,01 \\
\hline Mu_Orionis & $\mathrm{Aa}$ & 2,28 & 8.300 & 2,85 \\
\hline 3_Centauri & B8V & 2,47 & 9.638 & 2,8 \\
\hline Vela $X-1$ & B0.5Ib pulsar & 1,88 & 31.500 & $\sim 11,2$ \\
\hline HD_49798 & $\mathrm{sdO5.5}$ & 1,50 & 47.500 & 1,45 \\
\hline PSR J0348+0432 & pulsar & 2,01 & I & $13 \pm 2 \mathrm{~km}$ \\
\hline 14 Aurigae & white dwarf & 1,64 & 7.498 & / \\
\hline$G Q$ Lupi $b$ & planet & 1-36 MJup. & $2.650 \pm 100$ & Distance $100 \mathrm{AU}$ \\
\hline
\end{tabular}

Table 9. Star type /mass $\sim 2 /$ temperature and radius

The result of the two Sun masses is taken to exclude the discussions of the existence of different types of combustion that are created due to different star formations. [3] This is particularly expressed by the planet display, with temperatures of $2.650 \pm 100$, which is a star with an independent process of creating warmth and radiation. This is stressed in the table 4, with planets which temperatures are $\sim 2.700^{\circ} \mathrm{K}$ and their mass being from $12-24$ masses of Jupiter, and the star NML Cygni with its mass of 50 MSun and the temperature of $3.834^{\circ} \mathrm{K}$.

2.3. Bodies in distant orbits can be stars - planets

Table 10. Bodies with mass to 13 mass of Jupiter/temperature and distance

\begin{tabular}{|c|c|c|c|}
\hline Planet and Brown dwarf & Mass of Jup. & Temperature ${ }^{\circ} \mathrm{K}$ & Distance AU \\
\hline$H D 106906 b$ & $11 \pm 2$ & 1.800 & 120 \\
\hline $1 R X S 1609 b$ & $8(14)$ & 1.800 & 330 \\
\hline Cha 110913-773444 & $8(+7 ;-3)$ & $1.300-1.400$ & \\
\hline OTS 44 & 11,5 & $1.700-2.300$ & \\
\hline GQ Lupi b & $1-36$ & $2.650 \pm 100$ & 100 \\
\hline$R O X s 42 B b$ & 9 & $1.950 \pm 100$ & 157 \\
\hline$H D 44627$ & $13-14$ & $1.600-2.400$ & 275 \\
\hline DH Tauri b & 12 & 2.750 & 330 \\
\hline $2 M 1207 b$ & $4(+6 ;-1)$ & $1.600 \pm 100$ & 40 \\
\hline $2 M 044144$ & $9,8 \pm 1,8$ & 1.800 & $15 \pm 0.6$ \\
\hline 2MASS J2126-8140 & $13,3( \pm 1,7)$ & 1.800 & 6.900 \\
\hline$H R 8799 c$ & $7(+3 ;-2)$ & $1.090(+10 ;-90)$ & $\sim 38$ \\
\hline$H R 8799 d$ & $7(+3 ;-2)$ & $1.090(+10 ;-90)$ & $\sim 24$ \\
\hline HIP $65426 b$ & $9,0 \pm 3,0$ & $1.450 .0( \pm 150.0)$ & 92 \\
\hline
\end{tabular}

Table 10. Bodies with mass to 13 mass of Jupiter/temperature and distance

Table 10. eliminates the claims that objects below 13 masses of Jupiter can't have an independent production of high temperatures, which is measured also on stars $S$ Cassiopeiae 1.800; $W$ Aquilae 1.800; V Cyg 1.875; V384 Per 1.820; S Aur $1.940^{\circ} \mathrm{K}$. [8] 2.4. Observing the density of bodies in our system

Table 11. Rotation/density

\begin{tabular}{|c|c|c|c|c|c|c|}
\hline Body & Rotation & & $\begin{array}{l}\text { Mean } \\
\text { density } \mathrm{g} / \mathrm{cm}^{3}\end{array}$ & Mass Jupiter $=1$ & Magnetic field $\mathrm{G}$ & Type \\
\hline Sun & 25,38 & day & 1,408 & 1047 & $1-2$ (10-100 sunspots) & G2V \\
\hline Jupiter & 9,925 & hours & 1,326 & 1 & 4,2 (10-14 poles) & planets \\
\hline Saturn & 10,64 & hours & 0,687 & 0,299 & 0,2 & planets \\
\hline Uranus & $(-) 0,71833$ & day & 1,27 & 0,046 & 0,1 & planets \\
\hline Neptune & 0,6713 & day & 1,638 & 0,054 & 0,14 & Planets \\
\hline Sirius A & 16 & $\mathrm{~km} / \mathrm{s}$ & 0,568 & $2,063 \pm 0,023 \mathrm{M}_{\text {Sun }}$ & weak & $\mathrm{A} 0 \mathrm{~mA} 1 \mathrm{Va}$ \\
\hline PSR J1745-2900 & 3,76 & second & / & $1-3 \quad$ (mass Sun) & $10^{14}$ & pulsar \\
\hline
\end{tabular}

Table 11. Rotation/density

Here I will give an additional explanation for a claim that "A small star with a high mass will have a high density, because all of its mass is getting squeezed into a small space...hence, it's very dense. A larger star of the same mass will have a lower density due to its stuff not getting squeezed so much."[11] through 
the rotation of an object around its axis.

Jupiter has the fastest rotation in our system, but it doesn't affect the density of the planet - it is lower [4] than the one of Sun, Neptune and Pluto. Saturn is particularly interesting with its lowest density in the table 11. ( Pan 0,42 g/cm ${ }^{3}$, Atlas $0,46 \mathrm{~g} / \mathrm{cm}^{3}$, Pandora $0,48 \mathrm{~g} / \mathrm{cm}^{3}$, Prometheus $0,48 \pm 0,09 \mathrm{~g} / \mathrm{cm}^{3}$ 67P/Ch-G $0,533 \mathrm{~g} / \mathrm{cm}^{3}$, Amalthea $0,857 \pm 0,099 \mathrm{~g} / \mathrm{cm}^{3}$ are solid bodies).

This states that density doesn't change with the increase of mass, temperature and the speed of rotation. The speed of rotation in our system is significant with the objects that are inside the area, rich with matter, i.e., the area, where disks of gas and asteroid belts are created. The higher the frequency of matter incoming onto an object generally means that the discussed object will have a faster rotation and higher temperature.

Fast-rotating hot stars are generally situated in those parts of the space, which is rich with matter (nebulae).

Table 12. \% Mass of satellites Satellites /Central body

\begin{tabular}{lll}
\hline Body & $\begin{array}{l}\sim \% \text { Mass of satellites } \\
\text { Satellites /Central body }\end{array}$ & $\begin{array}{l}\text { Mean density } \\
\mathrm{kg} / \mathrm{m} 3\end{array}$ \\
\hline Pluto & 12,2 & 1750 \\
\hline Earth & 1,23 & 5515 \\
\hline Neptune & 0,385 & 1638 \\
\hline Sun & 0,14 & 1408 \\
\hline Saturn & 0,024 & 687 \\
\hline Jupiter & 0,021 & 1326 \\
\hline Uranus & 0,00677 & 1270 \\
\hline
\end{tabular}

Table 12. \% Mass of satellites Satellites /Central body

If only the influence of gravity on the objects in an orbit or in the correlation of two stars is exclusively measured, that would be a wrong thing to do and it is presented in table 12. Pluto is the smallest object and it has the biggest percentage of its satellites' mass in the relation an object's mass/its satellites' mass in the orbit.

The stars with a fast rotation create impressive systems, independently of their mass or radius, to the opposite of the stars with a slow rotation.
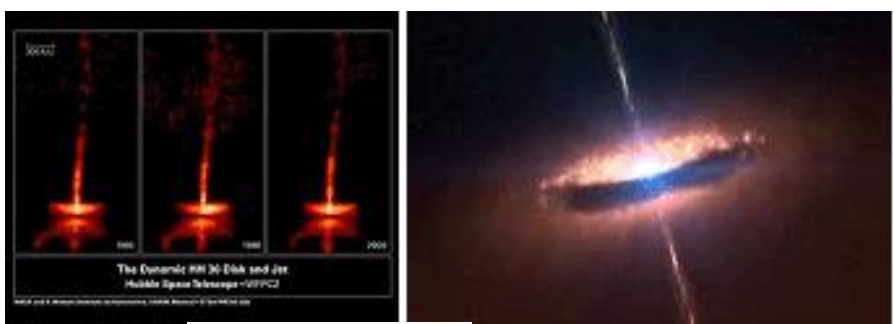

Figure 1. a fast rotating object

\subsection{The band of matter concentration and the influence of rotational speed on bodies in orbits and centers of galaxies}

In the formula for determining the behavior of planets, must be included temperatures of space and proximity to the central body, with special observation of the belt that is richer in matter.

Confirmation of this correctness it's easy to see that the satellites of Jupiter, Uranus, Neptune.. are in the zone where matter is concentrated. Their mass is significantly larger than other satellites.

It is obligatory to observe here reducing the distance of that belt, with shrinking temperatures of space as the planets move away from the central body, independent of the mass of the central body and the speed of rotation, though mass and the speed of rotation is and here very important.

Table 13. Orbital periods days, distance, mass

\begin{tabular}{|c|c|c|c|c|}
\hline Exoplanets & Mass Jup. & orbital periods days & Distance AU & $\begin{array}{l}B D+202457 c=1 \\
\text { orbital periods days }\end{array}$ \\
\hline$B D+202457 c$ & 12,47 & 621,99 & 2,01 & 1 \\
\hline$H D 213240 \mathrm{~b}$ & 4,5 & 951 & 2,03 & $+329,01$ \\
\hline$O G L E-2006-B L G-109 L b$ & 0,73 & $1.788,5$ & 2,3 & $+1.166,51$ \\
\hline Gliese $317 b$ & 2,5 & 692 & 1,5 & $+70,01$ \\
\hline$H D 95089 b$ & 1,2 & 507 & 1,51 & $-114,99$ \\
\hline$H D 183263 b$ & 3,67 & 626,5 & 1,51 & $+4,5$ \\
\hline$H D 143361 b$ & 3,48 & $1.046,2$ & 1,98 & $+424,21$ \\
\hline$H D 5319 b$ & 1,76 & 641 & 1,6697 & $+19,01$ \\
\hline V391 Pegasi b & 3,2 & 1.170 & 1,7 & $+548,01$ \\
\hline
\end{tabular}

Table 13. Orbital periods days, distance, mass; BD + $202457 \mathrm{c}=1$

Table 13. shows that similar or identical distance of planets from their central object doesn't result with the same orbital period. This data is seriously undermining the idea of the uniformed reduction of the gravitational influence on the objects in our system and it shows that the speed of the objects in the orbit depends on mass as well as on the rotational speed of the central object and the mass of the objects in the orbit.

All these principles mentioned above are the same for the galactical centers, which are the largest objects in the Universe. 
Table 14. galaxies, relationship: type galaxies / rotational speed of galaxies

\begin{tabular}{lll}
\hline Galaxies & Type galaxies & Speed of galaxies \\
\hline Fast-rotating galaxies & & \\
\hline$R X J 1131-1231$ & quasar & $\begin{array}{l}\text {,X-ray observations of RX J1131-1231 (RX J1131 for short) show it is whizzing around at } \\
\text { almost half the speed of light. [22] [23] }\end{array}$ \\
\hline Spindle galaxy & elliptical galaxy & ,possess a significant amount of rotation around the major axis“ \\
\hline NGC 6109 & Lenticular Galaxy & Within the knot, the rotation measure is $40 \pm 8$ rad m-2 [24] \\
\hline Contrary to: Slow Rotation & & \\
\hline UnC 12591 & spiral galaxy & maximum value of 225 kilometers per second \\
\hline Milky Way & spiral galaxy & the highest known rotational speed of about 500 km/s, \\
\hline Table 14. galaxy & spiral galaxy & $210 \pm 10(220$ kilometers per second Sun)
\end{tabular}

Table 14. galaxies, relationship: type galaxies / rotational speed of galaxies; No 1-3 Fast-rotating galaxies, No 4-6

Slow-rotating galaxies. From [10]

\section{Conclusion}

When there is an increase of data quantity in the database, the preconditions are created to discuss the white dwarfs within realistic values as small, fastrotating stars with the density, which is similar to other, both medium and large, hot stars. Small fastrotating stars (white dwarfs, pulsars, neutron stars, Wolf-Rayet stars, proto stars) have gas disks or significant asteroid belts, because they are formed inside the space, rich with matter. [7]

Very fast rotation of the central body creates fast orbits of gas, small and large objects.

With the constant increase of matter [9], a star gathers it from the orbits (including the process of migration of hydrogen and helium from the smaller objects towards a star [12]) and, because of growth, disks and asteroid belts are growing smaller, accordingly to the relation of: a star's mass/the mass of matter in its orbit.

Due to high temperatures of the fast-rotating stars, matter disintegrates into hydrogen (some helium is the product of the process of constant joining of particles). The traces of complex elements on hot objects are detected because there is a constant daily influx of matter, within which there are complex elements and compounds.

The speed of rotation with the increase of an object's mass affects more the level of temperature, because more quantity of mass gives an object a more complex structure, higher values of matter mixture and the creation of higher forces of pressure and friction. A higher value of particle work and a higher quantity of work, due to rotation, binary effects,... make the difference between cold and hot stars. When binary effects, made by the activity of gravity (the attraction force of matter), are ruled out, the rotation speed of an object determines the speeds of gas orbits and objects, with the remark that every object has an area in which matter is concentrated. Masses of the objects in that area are larger than masses of the objects in the orbit and therefore gas, dust and asteroids (disks and asteroid areas) are concentrated in such areas. [13], [14], [15], [16]

\section{Reference:}

1. 272 linnks type $R X J 1131-1231$; HD 183263 b; Jupiter; $G Q$ Lupi b; dist. $330 \mathrm{AU} ; \mathrm{BI} 253$ etc. in one to multiple steps leads to the source

2. https://astronomy.stackexchange.com/questions/13644/howdo-star-densities-work How do star densities work?

3. https://sciencing.com/calculate-stellar-radii-7496312.html How to Calculate Stellar Radii

4. https://imagine.gsfc.nasa.gov/science/objects/dwarfs $2 . h t m l$ „White Dwarf Stars“ Last Modified: December 2010

5. http://cds.cern.ch/record/435428/files/0004317.pdf "The Properties of Matter in White Dwarfs and Neutron Stars" Shmuel Balberg and Stuart L. Shapiro* Department of Physics, University of Illinois at Urbana-Champaign, 1110 W. Green St., Urbana, IL 61801

6. https://www.universetoday.com/24681/white-dwarf-stars/ February 4, 2009 by fraser cain, ,White Dwarf Stars“

7. https://www.ijsciences.com/pub/pdf/V82019021908.pdf „Effects of Rotation Araund the Axis on the Stars, Galaxy and Rotation of Universe" 3.4 . The density of smaller objects and stars, W.Duckss

8. https://arxiv.org/pdf/1601.07017.pdf „Constraints on the $\mathrm{H} 2 \mathrm{O}$ formation mechanism in the wind of carbon-rich AGB stars?" R. Lombaert1, 2 , L. Decin2, 3 , P. Royer2 , A. de Koter2, 3 , N.L.J. Cox2 , E. González-Alfonso4, D. Neufeld5 , J. De Ridder2 , M. Agúndez6 , J.A.D.L. Blommaert2, 7 , T. Khouri1, 3 , M.A.T. Groenewegen8 , F. Kerschbaum9, J. Cernicharo6 , B. Vandenbussche2, and C. Waelkens2 1

9. https://cordis.europa.eu/project/rcn/102627/reporting/en Cos mic Dust in the Terrestrial Atmosphere

10. http://www.sciencepublishinggroup.com/journal/paperinfo?jo urnalid=301\&doi=10.11648/j.ajaa.20180603.13 2.2. The effects of the stars' speed of rotation W.D.

11. https://scienceatyourdoorstep.com/2018/06/13/star-mass-anddensity/ Star Mass and Density june 13, 2018 / Emma

12. http://www.IntellectualArchive.com/files/Duckss.pdf „Why do Hydrogen and Helium Migrate" the Intellectual Archive W.D.

13. https://en.wikipedia.org/wiki/Moons_of_Jupiter\#List Io, Europa, Ganymede, Callisto

14. https://en.wikipedia.org/wiki/Moons_of_Saturn\#List Rea Titan, Hyperion, Iapetus

15. https://en.wikipedia.org/wiki/Moons_of_Neptune\#List Proteus, Triton, Nereid

16. https://en.wikipedia.org/wiki/Moons_of_Uranus\#List Miranda, Ariel, Umbriel, Titania, Oberon 